\title{
Die Geisteswissenschaften und ihr Bildungsdiskurs Zur Kartierung eines vernachlässigten Gebiets der Wissenschaftssoziologie
}

\section{The German Humanities and the Discourse of Bildung Toward Mapping a Neglected Territory within the Sociology of Science}

\section{Julian Hamann}

Universität Bonn, Forum Internationale Wissenschaft

Zusammenfassung: Der Beitrag rekonstruiert die diskursive Konstruktion geisteswissenschaftlicher Bildungsbegriffe und den Wandel des geisteswissenschaftlichen Feldes seit Beginn des 19. Jahrhunderts. Die Untersuchung zeigt anhand geisteswissenschaftlicher Selbstbeobachtungen, wie die diskursive Konstruktion von „Bildung“ einerseits auf Veränderungen des sozialen Feldes reagiert, in das der Bildungsdiskurs eingebettet ist, andererseits aber auch über Beharrungskräfte gegenüber sozialem Wandel verfügt. Die historisch stabilen Topoi einer dezidiert „freien“ und „wissenschaftlichen“ Bildung sind Elemente einer Positionierungsstrategie, die der akademischen Distinktion der Geisteswissenschaften dient. Der Artikel trägt zu einem soziologischen Verständnis der Geisteswissenschaften bei, das angesichts der einseitigen Fokussierung der Wissenschaftssoziologie auf die Natur- und Technikwissenschaften ein Desiderat darstellt.

Schlagworte: Soziologie der Sozial- und Geisteswissenschaften; Soziologie wissenschaftlichen Wissens; Diskursanalyse; Feldanalyse; Distinktion; Bildung.

Summary: This contribution reconstructs the discursive construction of the humanistic notion of "Bildung" and the transformation of the field of the German humanities since the beginning of the $19^{\text {th }}$ century. On the basis of academic self-reflections within the humanities, the analysis investigates how the discursive construction of "Bildung" is responsive to changes in the broader social field in which the discourse is embedded. At the same time, this discourse shows a remarkable persistence against social change. The historically stable topoi of a decidedly "free" and "scientific" "Bildung" are elements of a strategy of academic positioning that facilitates distinction in favor of the humanities. In light of the rather lopsided focus on natural and technical sciences within the sociology of science, this paper addresses a research gap by contributing to a sociological understanding of the German humanities.

Keywords: Sociology of the Social Sciences and Humanities; Sociology of Scientific Knowledge; Discourse Studies; Field Analysis; Distinction; Bildung. 
These are proofs of the final publication that is available at de Gruyter/Lucius \& Lucius via Zeitschrift für Soziologie, http://www.zfs-online.org/index.php/zfs/article/viewFile/3208/2745

\section{Einleitung: Die Geisteswissenschaften als wissenschaftssoziologischer Untersuchungsgegenstand}

Wer oder was sind die „Geisteswissenschaften“ aus soziologischer Perspektive? Worin ist ihre Krisenaffinität begründet? Warum scheinen sie sich mit Nachweisen gesellschaftlicher Relevanz und dem Drittmittelimperativ schwerer zu tun als andere Disziplinengruppen? In welchem Verhältnis stehen holistische Selbstverständnisse „der Geisteswissenschaften“ zu einer sich immer stärker spezialisierenden Fachkommunikation? Obwohl diese Fragen von hoher Aktualität sind und auf einen genuin wissenschaftssoziologischen Untersuchungsbereich abzielen, gibt es in der Wissenschaftssoziologie nur sehr vereinzelte Anstrengungen, auf den sich daraus ergebenden Forschungsbedarf zu reagieren. Entsprechende Klagen über eine einseitige Konzentration auf die Natur- und Technikwissenschaften finden sich in der Wissenschaftssoziologie seit Jahrzehnten, verhallen aber weitgehend ungehört (vgl. nur Bourdieu 1975: 24; Heintz 1993: 546; Kneer \& Moebius 2010: 10). Der vorliegende Beitrag adressiert dieses Desiderat im Sinne einer historisch informierten Wissenschaftssoziologie der Geisteswissenschaften. Dabei geht er aus diskursanalytischer Sicht davon aus, dass der Begriff „Geisteswissenschaften“ keine fest umrissene Einheit von Disziplinen bezeichnet, sondern als diskursives Label laufend über zuweilen konfliktreiche Selbstbeobachtungen aktualisiert wird. Eine feldanalytische Heuristik bezieht den sozialen Wandel des Kontextes ein, in den dieser selbstreflexive Diskurs eingebettet ist.

Ein Motiv der konstanten Selbstreflexion der Geisteswissenschaften sind ihre Bildungsbegriffe. Auseinandersetzungen über legitime Verständnisse von „Bildung“ sind seit über 200 Jahren eine zentrale Referenz in der geisteswissenschaftlichen Selbstbeobachtung. Die Beiträge erstrecken sich von einer Theorie der Bildung des Menschen (Humboldt 1960) über eine Theorie der Halbbildung (Adorno 2006) bis zu einer Theorie der Unbildung (Liessmann 2006). Für ein wissenschaftssoziologisches Verständnis der Disziplinengruppe gilt es, den historischen Verlauf dieser diskursiven „Bildung“ der „Geisteswissenschaften“ zu rekonstruieren und damit zu einer Kartierung der Geisteswissenschaften beizutragen.

Der Artikel schließt damit an einen noch überschaubaren wissenschaftssoziologischen Forschungsstand zu den Geisteswissenschaften an, der sich an seinen Rändern mit intellektuellensoziologischen und geschichtswissenschaftlichen Perspektiven sowie mit die Sozialwissenschaften einbeziehenden Studien überschneidet (vgl. dazu auch Dayé 2014). Fünf relevante Forschungsstränge lassen sich identifizieren: 1.) Viel Aufmerksamkeit haben insbesondere spezifische Mechanismen und Praktiken der symbolischen (Re-)Produktion erfahren (Kauppi 1996; Guetzkow et al. 2004; Steinmetz 2005; Angermüller 2007; Beer \& Koenig 2009; Lamont 
These are proofs of the final publication that is available at de Gruyter/Lucius \& Lucius via Zeitschrift für Soziologie, http://www.zfs-online.org/index.php/zfs/article/viewFile/3208/2745

2009; Brownley 2012). Der Bildungsdiskurs als Ort der Selbstreflexion (vgl. Bollenbeck 1994; Steenblock 1999) und als spezifische Form der symbolischen (Re-)Produktion war dabei aber bisher noch nicht Gegenstand einer soziologischen Analyse (vgl. Hamann 2011). 2.) Thematisiert worden sind auch Unterschiede und Gemeinsamkeiten zu den Sozial- und Naturwissenschaften (Sarton 1953; Lepenies 1985; Nowotny 2005; Krebs et al. 2005; Kagan 2009; Real 2012). Diese können jedoch noch besser erfasst werden, wenn die Geisteswissenschaften als eigenständiger Untersuchungsgegenstand konturiert werden und ihren analytischen Platz neben anderen Disziplinengruppen erhalten (vgl. Angermüller 2010; Camic et al. 2011). 3.) Für die Einbettung der Geisteswissenschaften in ihren jeweiligen nationalstaatlichen Kontext (vgl. Shumway 1994; Collins 2000; Sapiro 2003; Hollinger 2006; Behrens et al. 2010) hat die Definition und Pflege eines gesellschaftlichen Bildungskanons eine zentrale Bedeutung (vgl. Ringer 1989; Guillory 1993). 4.) Aus dieser Einbettung ergeben sich Ambivalenzen gesellschaftlicher Verwertungsansprüche (Kernan 1997; Donoghue 2008; Benneworth \& Jongbloed 2010; Böhler \& Maasen 2011; Kaldewey 2013; Gengnagel \& Hamann 2014), die sich dezidiert auch auf das universitäre Bildungsangebot beziehen (vgl. Kellermann 2006; Teichler 2011). 5.) Schließlich ist die Allgegenwärtigkeit geisteswissenschaftlicher Krisendiagnosen (vgl. nur Plumb 1964; Nussbaum 2010; Reinalter 2011; Jay 2014) Gegenstand soziologischer Forschung (Hall 1990; Ringer 1990; Weingart et al. 1991; Bourdieu 1992; Readings 1999). Dabei ist noch nicht ausreichend auf den Bildungsdiskurs als eine Referenz geisteswissenschaftlicher Selbstreflexionen rekurriert worden, die weiter als die Krisendiskurse zurückreicht und diese in Teilen erklären kann (vgl. Ringer 1989; Hamann 2014).

Im Sinne einer Genealogie geisteswissenschaftlicher Bildung zeigt der vorliegende Artikel, wie Bildungsverständnisse im Zeitverlauf variieren und auf den Wandel des Diskurskontextes verweisen (3.). Gleichzeitig kann aber auch ein gegenüber tief greifenden sozialen Umwälzungen der letzten 200 Jahre stabiler Kern der Bildungsbegriffe freigelegt werden, der sich in Abgrenzung zum gesellschaftlichen und akademischen Gegenüber konturiert (4.). Am Beispiel der eingangs skizzierten aktuellen Fragestellungen wird schließlich gezeigt, inwiefern von den gewonnenen Einsichten ein soziologisches Verständnis der Geisteswissenschaften ausgehen kann (5.). Die Kartierung geht von einem Zusammenspiel zwischen diskursiven Dynamiken und sozialem Wandel aus, für dessen Rekonstruktion eine Verknüpfung feld- und diskursanalytischer Heuristiken besonders geeignet ist. Im Folgenden werden dieser Zugang und seine methodische Umsetzung vorgestellt. 
These are proofs of the final publication that is available at de Gruyter/Lucius \& Lucius via Zeitschrift für Soziologie, http://www.zfs-online.org/index.php/zfs/article/viewFile/3208/2745

\section{Zu einer feld- und diskursanalytischen Genealogie der Geisteswissenschaften}

Geisteswissenschaftliche Selbstbeobachtungen finden sich in zahlreichen programmatischen Manifesten und historischen Verortungen. Weil die eigene Geschichte den Geisteswissenschaften eine zentrale Projektionsfläche für die jeweils gegenwärtige soziale Wirklichkeit darstellt, ist eine weit ausgreifende historische Perspektive notwendig. Ihr Startpunkt ist die Wende zum 19. Jahrhundert. Dies ist die Zeit, in der Wissenschaft programmatisch von ihrer außerwissenschaftlichen Anwendung getrennt und akademische Freiheit zur Grundlage von Forschung wird, sie materielle Unabhängigkeit von politischen oder religiösen Einflussversuchen gewinnt, ein modernes System wissenschaftlicher Disziplinen entsteht und Schule und Universität klar voneinander abgegrenzt werden (Turner 1980; Stichweh 1994). Im Rahmen dieses Akademisierungsprojekts bilden sich auch die modernen Geisteswissenschaften heraus (vgl. Bod et al. 2014).

In dem von diesem historischen Punkt bis in die Gegenwart reichenden Zeitraum verortet die Untersuchung vier Phasen, die aus dem empirischen Material geisteswissenschaftlicher Selbstverständigungen abgeleitet wurden. In jenen Phasen werden traditionelle Verständnisse von Bildung herausgefordert und verteidigt, so dass zu anderen Zeitpunkten implizit bleibende Annahmen expliziert und für die Analyse greifbar werden. Die Konstruktion neuer Bildungsbegriffe ist besonders konfliktreich von 1793 bis 1821, wo die Konstitution eines modernen geisteswissenschaftlichen Selbstverständnisses stattfindet, das auch auf spezifischen Bildungsbegriffen fußt; von 1872 bis 1925, wo sich die Geisteswissenschaften auch mit ihrem Bildungsverständnis gegenüber den immer dominanter werdenden Naturwissenschaften und im entstandenen einheitlichen Nationalstaat positionieren müssen; von 1945 bis 1968, wo restaurative Tendenzen nicht nur im geisteswissenschaftlichen Bildungsdiskurs durch die einsetzende Bildungsexpansion schnell unhaltbar werden; und von 1986 bis 2012, wo ein gesellschaftliches Interesse an wissenschaftlichen Leistungen und Relevanzen expliziter geäußert wird und auch geisteswissenschaftliche Bildungsbegriffe herausfordert.

Die in diesen Phasen besonders konfliktreiche Konstruktion von Bildungsbegriffen ist in Anlehnung an eine auch als interpretative Analytik bezeichnete diskursanalytische Heuristik (Dreyfus \& Rabinow 1987; Diaz-Bone 2005; Keller 2005) aus dem empirischen Material rekonstruiert worden. Das aus selbstreflexiven Manifesten und Vergewisserungen über „Bildung“" zusammengestellte Textkorpus besteht aus insgesamt 3.202 Seiten, die 119 Beiträge von 77 Autoren umfassen. Dieses Material wurde unter Verwendung qualitativer Datenanalysesoftware in mehreren Durchgängen thematisch indiziert (zum theoriegenerierenden Codieren vgl. Strauss \& Corbin 1996; siehe auch Friese 2012). In dem mehrstufigen Prozess wurden 3.599 
These are proofs of the final publication that is available at de Gruyter/Lucius \& Lucius via Zeitschrift für Soziologie, http://www.zfs-online.org/index.php/zfs/article/viewFile/3208/2745

Textstellen codiert. Die dabei entwickelte Struktur von insgesamt 58 Codes auf fünf inhaltlichen Dimensionen (vgl. Tab. 5 im Online-Anhang unter www.zfs-online.org, zur Rekonstruktion einer mehrdimensionalen „Phänomenstruktur“ vgl. Keller 2008)gestattet es, in den Texten materialisierte Sprechakte mit Bezug auf den Bildungsbegriff als diskursiv formierte Aussagen zu begreifen und aus ihrer jeweiligen Verteilung auf systematische Formationsregeln zu schließen. Der Bildungsdiskurs wird so als ein Regelsystem sichtbar, das begriffliche Ausprägungen von „Bildung“ formiert, indem es entsprechende Aussagen strukturiert und das gleichzeitig von dieser diskursiven Praxis erst hervorgebracht wird (vgl. Foucault 1981, 1996). Dieses interpretativ-rekonstruktive und eng am empirischen Material orientierte Vorgehen erscheint besonders geeignet dafür, die Geisteswissenschaften über ihren Bildungsdiskurs zu kartieren.

Die anhand der codierten Textstellen rekonstruierten geisteswissenschaftlichen Bildungsbegriffe sind, darauf kann bereits mit Foucault (1996: 179) hingewiesen werden, kein „von irgendwoher kommender, von selbst entstandener Prozeß“, sie sind mitnichten „ohne jeglichen historischen Kontext“, sondern vielmehr „eine »Antwort« auf eine konkrete Situation, die durchaus real ist.“ Doch wie ist der „historische Kontext“, von dem Foucault spricht, analytisch zu fassen? Die Annahme, dass diskursive Konstruktionen an spezifische Diskurskontexte andocken, legt nahe, dass es sich bei den Begriffen „Geisteswissenschaften“ und „Bildung“ um mehr handelt als um diskursive Effekte. Sie sind nicht nur durch eine generative diskursive Praxis konstruiert, sondern weisen als Funktion sozialer Kräfteverhältnisse auch über den Diskurs hinaus. Zu diesen Kräfteverhältnissen zählen beispielsweise Studierendenzahlen, Drittmittelförderung, aber auch Auseinandersetzungen um legitime Forschungsgegenstände und -methoden, Kanonisierungsbefugnisse und -erwartungen. Weil diskursive Dynamiken besser verständlich werden, wenn der soziale Wandel des Diskurskontextes in den Analyserahmen integriert wird, muss ein gegenstandsadäquater Zugang diesen Produktions- und Rezeptionskontext des Bildungsdiskurses berücksichtigen.

Foucaults Diskursanalyse verfügt zwar über ein grundsätzliches Bewusstsein für den Stellenwert von Diskurskontexten, für ihre Untersuchung stehen jedoch kaum heuristische Instrumente bereit, die den Ansprüchen empirischer Sozialforschung genügen. Über ein in dieser Hinsicht ausgearbeitetes Repertoire verfügt dagegen Bourdieus Feldanalyse, in deren Heuristik die Rekonstruktion des Bildungsdiskurses daher eingebettet wird. Im Weiteren wird der Produktions- und Rezeptionskontext des Bildungsdiskurses näherungsweise über materielle und symbolische Kräfteverhältnisse im geisteswissenschaftlichen Feld erfasst. Um eine diachrone Vergleichbarkeit zu ermöglichen, erfolgt die Bestimmung dafür relevanter Kontextfaktoren 
These are proofs of the final publication that is available at de Gruyter/Lucius \& Lucius via Zeitschrift für Soziologie, http://www.zfs-online.org/index.php/zfs/article/viewFile/3208/2745

schematisch. Theoriegeleitet und orientiert an bestehenden Analysen wissenschaftlicher Felder (Bourdieu 1992; Karabel 2006; Angermüller 2007; Münch 2007; Maeße 2013) werden sozialstrukturelle Merkmale der studentischen Population sowie die institutionelle Fächerstruktur und die Drittmittelverteilung als Faktoren für die materielle Struktur des Feldes als Diskurskontext herangezogen. Dominante Verständnisse legitimer (Geistes-)Wissenschaft sowie Zuschreibungen zur Situation und Position der Professorenschaft als Trägerin solcher Wissenschaftsverständnisse ermöglichen ein Verständnis der symbolischen Struktur des geisteswissenschaftlichen Feldes als Diskurskontext. Über die jeweiligen Ausprägungen der diskursrelevanten materiellen und symbolischen Kräfteverhältnisse informieren die einschlägige Primär- und Sekundärliteratur aus der Hochschulforschung, der Wissenschaftsgeschichte und -soziologie sowie Sekundäranalysen von Statistiken.

Die hier vorgenommene Verknüpfung feld- und diskursanalytischer Perspektiven hat sich aufgrund gemeinsamer strukturationstheoretischer und methodologischer Grundannahmen bereits als ertragreich erwiesen (vgl. nur Diaz-Bone 2002; Angermüller 2007; Maeße 2010; Schmidt-Wellenburg 2013; Hamann 2014). Der analytische Zugang adressiert Schwachstellen beider Perspektiven: Während die Diskursanalyse für die Einbettung ihres Gegenstands in spezifische (Feld-)Kontexte sensibilisiert wird, kann die von Feldanalysen vertretene Annahme einer Homologie von Positionierungen und Positionen mit diskursanalytischem Instrumentarium empirisch überprüft werden. Was über geisteswissenschaftliche Bildung gesagt werden kann und was an der Oberfläche der Bildungsdiskurses als inhaltliche Ausprägung von „Bildung“ erscheint, wird demgemäß als diskurs- und feldlogisches Zusammenspiel diskursiver Strukturierungen mit geisteswissenschaftlichen Diskurskontexten begriffen.

\section{Der Wandel geisteswissenschaftlicher Bildungsbegriffe von Preußen bis zur Berliner Republik}

Die Analyse der Zusammenhänge zwischen dem Bildungsdiskurs und seinen feldspezifischen Kontexten illustriert zunächst, wie sensibel der Diskurs auf sozialen Wandel reagiert. Soweit dies der Fall ist, kann davon ausgegangen werden, dass die diskursiven Formationsregeln über eine relativ geringe Eigenlogik gegenüber den Kräfteverhältnissen verfügen, in die sie eingebettet sind. Empirisch stehen dann Veränderungen inhaltlicher Ausprägungen von Bildungsbegriffen in einem Verweisungszusammenhang mit dem Wandel der materiellen und symbolischen Strukturen der jeweiligen Diskurskontexte. Um dieses Passungsverhältnis nachvollzie- 
These are proofs of the final publication that is available at de Gruyter/Lucius \& Lucius via Zeitschrift für Soziologie, http://www.zfs-online.org/index.php/zfs/article/viewFile/3208/2745

hen zu können, werden im Folgenden zwei zentrale inhaltliche Dimensionen geisteswissenschaftlicher Bildungsbegriffe fokussiert: Zuschreibungen bestimmter (1.) Zwecke und Funktionen sowie (2.) praktischer Formen und Bedingungen von Bildung.

\subsection{Charakterbildung durch den Kathedervortrag und die Rhetorik sozialer Inklusivität: Bildungsdiskurs und sozialer Wandel in Preußen (1793-1821)}

Im Umfeld der preußischen Universitätsreform wird geisteswissenschaftlicher Bildung der Zweck zugeschrieben, zu einer universellen und ganzheitlichen Höherentwicklung des Charakters beizutragen. Bildung hat, so hält Fichte (1845: 332) stellvertretend für seine Zeitgenossen fest, die Funktion der „sittlichen Veredlung des ganzen Menschen“ (vgl. Hegel 1907: 320; Humboldt 1956: 379; 1960). Republikanische Deutungen wie die von Fichte (z.B. 1845: 328330) oder Steffens (1910: 233, 243) legitimieren diese Funktionszuschreibung mit den Bedürfnissen des Staates und stehen damit jenen Positionen gegenüber, für die Charakterbildung ihren Zweck in sich selbst trägt (vgl. Schleiermacher 1956: 231, 301). Praktisch soll sich eine solche ganzheitliche Veredelung des Charakters erstens in der Vorlesung realisieren. Während Seminare als auf das Spezielle und Besondere gerichtet gesehen werden (vgl. Schleiermacher 1956: 252, 264-265), vollziehe sich der Forschungs- und Erkenntnisprozess gerade im Kathedervortrag, dem „freie[n] mündliche[n] Vortrag vor Zuhörern“ (Humboldt 1956: 382), jedes Mal aufs Neue und übertrage sich so auf die Zuhörer (vgl. Schlegel 1971: 60-61; Krug 1805). Als legitime Praxisform geisteswissenschaftlicher Bildung gilt zweitens auch die sozial inklusive Rekrutierung der Bildungssubjekte. Nach diesem Verständnis richtet sich Bildung prinzipiell an die ganze preußische Nation, sie muss ,allen angeboten [...] werden, damit man sicher sei, daß nirgends dieses seltne Talent, aus Mangel an Kunde seiner, ungebraucht verloren gehe" (Fichte 1910: 20; vgl. 1845: 324; Schleiermacher 1956: 276-278; Humboldt 1964: 218). Als dominante Ausprägungen des Bildungsbegriffes können demnach die Funktion der universellen Charakterbildung und die Praxisformen der Vorlesung und der sozial inklusiven Rekrutierung rekonstruiert werden.

Die allgemeineren Voraussetzungen dieser inhaltlichen Ausprägungen liegen in der Staatsräson des preußischen Kulturstaats. Dieser profitiert von der umfassenden Charakterbildung zukünftiger Beamter, während sein auf dem Boden der kantischen Philosophie fußendes Reformprogramm der sozial inklusiven Bildungsrhetorik ihre moralisch-politische Grundlegung verleiht. Der feldspezifische Diskurskontext, mit dem die skizzierten Ausprägungen geisteswissenschaftlicher Bildung korrespondieren, ist symbolisch strukturiert durch ein idealistischneuhumanistisches Wissenschaftsverständnis, das als überaus wirkmächtiger Wissensbestand 
These are proofs of the final publication that is available at de Gruyter/Lucius \& Lucius via Zeitschrift für Soziologie, http://www.zfs-online.org/index.php/zfs/article/viewFile/3208/2745

einen Produktions- und Rezeptionskontext des Bildungsdiskurses darstellt, dem viele seiner Autoren zugerechnet werden können (vgl. nur Fichte 1997; Schelling 1830; Hegel 1907). So besteht eine Passung zwischen den neuhumanistischen Auffassungen legitimer Wissenschaft und einem Begriff der ganzheitlichen Charakterbildung, der in der Praxis inklusiv allen Menschen zugutekommen soll. Idealistische Elemente des Wissenschaftsverständnisses finden sich wiederum darin wieder, dass die umfassenden Wissensinhalte, von denen der Idealismus ausgeht, nicht in auf das Besondere gerichteten Seminaren, sondern in der Vorlesung ihre geeignete Vermittlungspraxis finden. Neben dem Wissenschaftsverständnis bilden Wissensbestände über die Situation der Professorenschaft weitere symbolische Kräfteverhältnisse, für die der Bildungsdiskurs sensibel ist. Akademiker wachsen an der Wende zum 19. Jahrhundert in eine dominante Stellung im sozialen Raum hinein, die vor allem auf ihrem kulturellen Kapital beruht (Klinge 2004: 120). Vor dem Hintergrund des Wissens über diese spezifische Situation verschleiert die Anrufung einer inklusiven Bildungspraxis ohne soziale Vorbedingungen die sozialstrukturellen Grundlagen von Bildung und damit auch der eigenen sozialräumlichen Dominanz. Die Rekrutierungsmechanismen, die sowohl der professoralen als auch der studentischen Population zugrunde liegen, werden stattdessen mit einem „Naturzustande der Gelehrsamkeit“ (Kant 2005: 16) verklärt. Professoren werden außerdem als umfassend gelehrte Persönlichkeiten gesehen (Ben-David 1971: 122; Brandt 2001: 132-133). Die Betonung ganzheitlicher Charakterbildung schließt an diese Behauptung an und erscheint in diesem Lichte als zentraler Mechanismus der akademischen Habitusformation.

Der Bildungsdiskurs verweist jedoch nicht nur auf symbolische Strukturen des Feldkontextes. Der Rhetorik einer sozial inklusiven Rekrutierungspraxis steht in materieller Hinsicht eine höchst selektive Zusammensetzung der geisteswissenschaftlichen Studierenden aus dem neu entstandenen Bildungsbürgertum gegenüber (Titze 1987: 27-30; Lundgreen 2000: 179), aufgrund derer im Studium bestimmte habituelle Dispositionen und Kenntnisse schon aus sozialstrukturellen Gründen vorausgesetzt werden können. Erst diese standesgemäße Sozialisation und der durch sie garantierte studentische Habitus geben der Behauptung Sinn, dass Bildung den Charakter zu veredeln habe. Des Weiteren kann eine Rhetorik der Inklusivität nur legitim sein, weil sie sich auf die faktische soziale Exklusivität des Studiums verlassen kann, weil sich die tatsächliche Bildungspraxis eben nicht mit den Folgen einer sozial inklusiven Zusammensetzung der Studierenden auseinandersetzen muss. In der zweiten Phase wird deutlich, dass diese inklusive Rhetorik aussetzt, sobald die Studierenden tatsächlich offener rekrutiert werden. 
These are proofs of the final publication that is available at de Gruyter/Lucius \& Lucius via Zeitschrift für Soziologie, http://www.zfs-online.org/index.php/zfs/article/viewFile/3208/2745

\subsection{Fachlich-methodische Berufsausbildung unter sozial exklusiven Bedingungen: Bildungsdiskurs und sozialer Wandel im Kaiserreich und der Weimarer Republik} (1872-1925)

Tatsächlich entwickelt sich angesichts der ersten Vorläufer der Bildungsexpansion im letzten Drittel des 19. Jahrhunderts recht bald ein Verständnis der praktischen Bedingungen von Bildung, das auf soziale Selektivität abhebt. So kann Weber (1922: 529) Bildung als ,geistesaristokratische Angelegenheit" charakterisieren, während sich die Forderung nach einer sozial exklusiven Bildungspraxis bei Jaeger (1960: 72-74) in der Klage ausdrückt, Bildung sei „ein Massenartikel geworden, billig und schlecht“ (vgl. Nietzsche 1954: 193; Scheler 1954: 16). Es ist bezeichnend, dass ausgerechnet der Neuhumanist Spranger (1909: 36) an einer inklusiven Rhetorik festhält, die ,alle Menschen zu Aristokraten zu machen strebt“. Als dominante Funktion geisteswissenschaftlicher Bildung erscheint nun nicht mehr allein die Charakterbildung (vgl. Jaeger 1960: 77-78; Scheler 1954: 23), sondern auch die methodische, fachlich-berufliche Ausbildung. Beispielhaft dafür ist Webers (1922: 453) Beschreibung wissenschaftlicher Bildung als „fachmäßige Schulung seitens fachmäßiger Qualifizierter“. Es wird zu einer wichtigen Funktion des Studiums, jene Fähigkeiten zu verleihen, derer es bedarf, „um den praktischen Aufgaben gerecht zu werden.“ (Zeller 1879: 5; vgl. Dilthey 1959: 21; Spranger 1909: 500) Dominante Ausprägungen des Bildungsbegriffes in dieser zweiten Phase sind demzufolge die Funktion der methodisch-fachlichen Berufsausbildung sowie die Anrufung einer sozial exklusiven Bildungspraxis.

Inwiefern verweisen diese Ausprägungen auf den Diskurskontext? Symbolisch ist das Feld von einem naturwissenschaftlich geprägten Forschungsklima beeinflusst, gegenüber dem sich dominante Wissenschaftsverständnisse in den Geisteswissenschaften bewähren müssen. Entsprechende Strategien versuchen entweder, der Philosophie eine Sonderstellung außerhalb des Gesamtkontextes empirisch vorgehender Disziplinen zuzuweisen (zu den Neukantianern vgl. Beiser 2008), oder den erkenntnistheoretischen Führungsanspruch auf den stärker empirie- und methodenorientierten Historismus zu übertragen (vgl. Jaeger \& Rüsen 1992). Wirkmächtig unternimmt schließlich auch Dilthey (1958: 117) den Versuch, „die selbständige Stellung der Geisteswissenschaften den Naturwissenschaften gegenüber darzutun“, indem er das subjektive Verstehen in die Technik der hermeneutischen Methode überführt. Bereits die schematische Darstellung dieser Auseinandersetzungen macht deutlich, dass empirische Forschung und elaborierte Methoden nun auch in den Geisteswissenschaften feste Bestandteile eines legitimen Wissenschaftsverständnisses sind. Es ist dieser Wandel der symbolischen Kräfteverhältnisse, 
These are proofs of the final publication that is available at de Gruyter/Lucius \& Lucius via Zeitschrift für Soziologie, http://www.zfs-online.org/index.php/zfs/article/viewFile/3208/2745

der sich im Bildungsdiskurs als Anrufung einer wissenschaftlich-methodischen Ausbildungsfunktion niederschlägt.

In materieller Hinsicht finden die inhaltlichen Ausprägungen geisteswissenschaftlicher Bildung ihre Voraussetzungen erstens in der institutionellen Differenzierung der Disziplinengruppe. So steigt die Zahl obligatorischer Kernfächer, die nun die Grundausstattung an geisteswissenschaftlichen Ordinaten ausmachen, während auch für bereits bestehende Fächer ein $\mathrm{Zu}$ wachs an Lehrstühlen zu verzeichnen ist (Lundgreen 1983: 149-163; Baumgarten 1997: 55-56; Charle 2004: 65). Durch diesen Wachstums- und Differenzierungsprozess erfahren disziplinäre Besonderheiten einen Bedeutungsgewinn. Mit der Zwecksetzung, Bildung habe spezifische Methodenkenntnisse und fachliche Kompetenzen zu vermitteln, schließt der Bildungsdiskurs an diesen Wandel an. Zweitens wird die materielle Feldstruktur ab dem letzten Drittel des 19. Jahrhunderts durch einen Anstieg der Studierendenpopulation charakterisiert - eine Entwicklung, mit der die allgemeine Angst akademischer Eliten vor „Vermassung“ einhergeht (vgl. Ringer 1990) und die ihre gesamtgesellschaftliche Entsprechung in den Modernisierungstendenzen des Kaiserreichs und der Weimarer Republik findet. Jene Öffnung der Rekrutierungsmechanismen zeigt sich für die Geisteswissenschaften nicht nur quantitativ im Vergleich zur Grundgesamtheit der Studierenden, sondern auch in ihrer vergleichsweise heterogenen sozialen Zusammensetzung (vgl. Busch 1977: 79; Titze 1987: 122-123, 140-141; Ringer 2004). Die im Bildungsdiskurs rekonstruierten Ausprägungen sind responsiv für diese Entwicklung. Zum einen schlägt sich die offenere Rekrutierung der Studierenden in einer Rhetorik der sozialen Exklusivität und in Klagen über Überfüllungstendenzen nieder. Zum anderen ist der hohe Stellenwert fachlich-beruflicher Kenntnisse dadurch bedingt, dass Bildung nun auf ein breiteres und hinsichtlich der sozialen Herkunft heterogeneres Publikum zielt, das nicht mehr vornehmlich an die Wissenschaft, sondern an verschiedene akademische Berufe vermittelt werden muss.

\subsection{Kritische Erkenntnis und akademische Abschlüsse trotz Vermassung:}

Bildungsdiskurs und sozialer Wandel in der Bonner Republik (1945-1968)

Nachdem in den Geisteswissenschaften die personell wie inhaltlich restaurativen Tendenzen der Nachkriegszeit überwunden sind, kristallisiert sich im Bildungsdiskurs die Zwecksetzung heraus, Bildung habe kritische Erkenntnis zu ermöglichen. Damit soll ein Beitrag zur politischen Erziehung geleistet, mitunter ein „Widerstand des Geistes [...] gegen die Verhältnisse“ ermöglicht werden (Adorno 1986: 335; vgl. Litt 1952: 6; Heimpel 1956: 17; Habermas 1969a: 203). Zum ersten Mal erhält Bildung außerdem die dominante Funktion, Studierende mit einem akademischen Abschluss zu versorgen. Paradigmatisch wird die „Ausbildung zur Fertigkeit für 
These are proofs of the final publication that is available at de Gruyter/Lucius \& Lucius via Zeitschrift für Soziologie, http://www.zfs-online.org/index.php/zfs/article/viewFile/3208/2745

einen bestimmten Beruf“ bei Jaspers (1946: 33) zu einem elementaren Moment geisteswissenschaftlicher Bildung, während Habermas (1969b: 117) fordern kann, dass Absolventen „mit den für die Ausübung einer akademischen Berufsrolle relevanten Eigenschaften und Einstellungen“" ausgestattet werden (vgl. Spranger 1961: 159; Heimpel 1956: 13; Habermas 1969c: 101-102). Den Funktionsbestimmungen kritischer Erkenntnis und akademischer Abschlüsse steht die praktische Bedingung der Vermassung gegenüber, die nun das dominante Motiv von Reflexionen über die Praxis der Bildung ist. Die Rede ist von einer „Überflutung“ (Anrich 1960: 58) und von „,nicht mehr zu bewältigenden Probleme[n] des Massenstudiums“ (Habermas 1969a: 236; vgl. Jaspers 1946: 57-58; Spranger 1961: 182; Heimpel 1956: 17-18; Adorno 2006: 21), die nicht selten dem nun unerreichbar scheinenden Ideal der Gelehrtengemeinschaft als „sokratische[r] Beziehung ohne Autorität, auf gleichem Niveau“ (Jaspers 1946: 46; vgl. Heimpel 1956: 21, 23; Anrich 1960: 31) entgegenstehen. Somit lassen sich dominante Funktionen kritischer Erkenntnis und akademischer Abschlüsse sowie eine Bildungspraxis der Vermassung rekonstruieren.

Was sind die Kontextbedingungen, auf die diese Ausprägungen des Bildungsbegriffes verweisen? Mit Blick auf weiter gefasste Prozesse sozialen Wandels stehen die im Diskurs rekonstruierten Klagen gegen eine Vermassung in engem Zusammenhang mit der Bildungsexpansion. Die Zwecksetzung, Bildung habe kritische Erkenntnis zu ermöglichen, steht im Einklang mit dem Klima der Demokratisierung und Liberalisierung in der Bundesrepublik. Im Feld selbst wirken legitime Wissenschaftsverständnisse als symbolische Kräfteverhältnisse auf den Bildungsdiskurs ein. Entsprechende Debatten zielen auf eine kritisch-emanzipative Politisierung der Disziplinen (zur Philosophie vgl. Adorno et al. 1972; zur Sprach- und Literaturwissenschaft Bundesassistentenkonferenz 1971; zur Geschichtswissenschaft Groh 1973) sowie auf eine reflexive Steigerung ihres analytischen Potenzials. Dies soll erreicht werden durch die Offenlegung theoretischer Axiome in legitimierenden Fachdiskursen (zur philosophischen Wissenschaftstheorie vgl. Schnädelbach 1990; zur Geschichtswissenschaft Mommsen 1971), durch empirisch gesicherte und formalisierte Beschreibungen (zur Analytischen Philosophie vgl. Baumgartner \& Sass 1979; zur Sprach- und Literaturwissenschaft Lakoff 1971; Chomsky 1973) oder durch gesamtgesellschaftliche Kontextualisierungen der Forschungsgegenstände (zur Sprach- und Literaturwissenschaft vgl. Jauß 1967; zur Geschichtswissenschaft Wehler 1980). An diese reflexiv politisierten Wissenschaftsverständnisse schließt der Bildungsdiskurs an, wenn kritische Erkenntnis als dominante Funktion geisteswissenschaftlicher Bildung erscheint. Auch die Orientierung an akademischen Abschlüssen kann verstanden werden als eine 
These are proofs of the final publication that is available at de Gruyter/Lucius \& Lucius via Zeitschrift für Soziologie, http://www.zfs-online.org/index.php/zfs/article/viewFile/3208/2745

diskursive Verarbeitung der neuen gesellschaftspolitischen Verantwortung, in der sich die Geisteswissenschaften nun sehen.

In materieller Hinsicht verändert sich der Diskurskontext ab den 1950er Jahren zum einen durch die Expansion der institutionellen Struktur. Davon profitieren insbesondere lehrerbildende Fächer wie Geschichtswissenschaft, Anglistik und Germanistik, in denen wiederum ein besonderes Gewicht auf didaktisch ausgewiesenen Denominationen liegt (Weingart et al. 1991: 248-251). Zu diesem durch Praxisdruck vorangetriebenen Wandel der materiellen Feldstruktur weist der Bildungsdiskurs eine Passung auf, weil geisteswissenschaftliche Bildung jetzt ebenso praxisorientiert an Abschlüssen ausgerichtet wird. Zum anderen verändern sich die materiellen Kräfteverhältnisse durch ein massives Wachstum der Studierendenpopulation. Insgesamt steigt die Zahl der Studierenden in den Kulturwissenschaften von 1954 bis 1978/79 um den Faktor 13,8 und damit stärker als die Gesamtzahl der Studierenden (Wachstum um den Faktor 11,4; (Statistisches Bundesamt 1955: 92, 94; 1980: 341). Mit Verzögerung sinkt dann auch die soziale Selektivität der Rekrutierungsmechanismen (Kath 1977). Im Diskurs schlagen sich die steigenden Studierendenzahlen in der Abschlussorientierung von Bildung nieder, mit der ihr die Verantwortung zugeschrieben wird, die gewachsene Studierendenschaft auf den Arbeitsmarkt vorzubereiten. Der Bildungsdiskurs reagiert aber vor allem dann auf die neuen Verhältnisse, wenn die praktischen Bedingungen geisteswissenschaftlicher Bildung mit Begriffen wie „Vermassung" beschrieben werden.

\subsection{Orientierung und Arbeitsmarktbefähigung unter Bedingungen der Normierung und} Verschulung: Bildungsdiskurs und sozialer Wandel in der Berliner Republik (19862012)

Die in den 1950er und 1960er Jahren bereits erkennbare Abschlussorientierung geisteswissenschaftlicher Bildung intensiviert sich ab Mitte der 1980er Jahre noch einmal. Zum ersten Mal ist die Zwecksetzung legitim, dass Bildung Studierende nicht nur mit Abschlüssen versorgen soll, sondern in einem umfassenden Sinne für ihre Arbeitsmarkttauglichkeit zu sorgen hat. So kann Herrmann (1989: 56) hervorheben, dass die Funktion der Geisteswissenschaften auch darin liege, ,die unumgängliche Qualifikation der für die hochindustrialisierte Gesellschaft notwendigen Arbeitskräfte zu sichern“. Geisteswissenschaftliche Bildung müsse, so positioniert sich nun eine ganze Reihe von Autoren, ,der gesamten Gesellschaft zur Ausbildung von Fachund Führungskräften dienen“ (Arnswald 2005: 154; vgl. Fabian 1996: 28; Rüsen 2005: 240; 
These are proofs of the final publication that is available at de Gruyter/Lucius \& Lucius via Zeitschrift für Soziologie, http://www.zfs-online.org/index.php/zfs/article/viewFile/3208/2745

Herbert 2009: 41). ${ }^{1}$ Noch dominanter als die Funktionsbestimmung der Arbeitsmarktbefähigung ist die Behauptung, Bildung habe die Gesellschaft an sich sowie das Individuum in der Gesellschaft zu orientieren. Bei Rüsen (2007: 400) wird der Bildung beispielhaft eine „kulturelle Orientierungsfunktion“ attestiert, die als „Sinnkompetenz“ nicht selten in der Tradition der Aufklärung formuliert und von einer bloßen Ansammlung von Wissen unterschieden wird (vgl. Marquard 1986: 110; Frühwald 1991: 80, 108; Liessmann 2006: 59; Brandt 2011: 169170). Die praktischen Bedingungen von Bildung scheinen nun durch Verschulung sowie durch bürokratische Normierung und Überformung bestimmt. Beklagt wird die Transformation der Universität in „schulähnliche Unterrichtsstätten“ (Gethmann et al. 2005: 21), die „unter der Ägide von Rechnungseinheiten der Bürokratie“ stehen (Brandt 2011: 166; vgl. Brenner 1997: 77; Liessmann 2006: 108-109). Dominante Ausprägungen des Bildungsbegriffes sind demnach die Funktionen der Arbeitsmarktbefähigung und der individuellen wie gesellschaftlichen Orientierung sowie praktische Bedingungen der Verschulung und bürokratischen Normierung.

Der feldspezifische Diskurskontext, auf den diese Ausprägungen verweisen, ist in symbolischer Hinsicht gekennzeichnet durch Wissensbestände über die Situation der Professorenschaft. Anekdoten und Selbstzuschreibungen (vgl. nur Simon 1989; Mittelstraß 2002: 75) lassen ebenso wie professionssoziologische Studien (Enders 1998: 59, 67; Enders \& Schimank 2001) darauf schließen, dass Professor/-innen gerade in den Geisteswissenschaften durch ihren Autonomieverlust und gesunkene Definitionsmacht charakterisiert sind. Im Bildungsdiskurs wird diese Behauptung durch Klagen über normierte und fremdbestimmte Praxisformen von Bildung verarbeitet. Weiterhin ist das Feld symbolisch durch Auseinandersetzungen strukturiert, die Fragen nach der gesellschaftlichen Bedeutung der Geisteswissenschaften in den Vordergrund rücken. In einem breiteren akademischen Kontext kann dieses „Ringen um Relevanz“ (Böhler \& Maasen 2011: 64) zurückgeführt werden auf neu geschaffene Markt- und Wettbewerbsstrukturen (vgl. Wissenschaftsrat 1985; Hochschulrektorenkonferenz 1992). Strittig ist in diesen Debatten über ein legitimes Wissenschaftsverständnis, worin die gesellschaftliche Bedeutung der Geisteswissenschaften bestehen könnte und wie nach ihr gefragt werden darf (vgl. nur Marquard 1986; Schnädelbach 1988; Gauger \& Rüther 2007; Heidbrink \& Welzer 2007). Ihre Entsprechung im Bildungsdiskurs findet die Frage nach der gesellschaftlichen Bedeutung der Geisteswissenschaften, wenn Bildung durch ihre besondere Orientierungsfunktion und ihre Arbeitsmarktbefähigung in Wert gesetzt wird.

1 Auffallend ist, dass das Bildungssubjekt, auf das sich diese Orientierung beruft, jetzt nicht mehr der „Student“, sondern der „Absolvent“ ist (vgl. nur Gethmann et al. 2005: 25; Nida-Rümelin 2005: 25). 
These are proofs of the final publication that is available at de Gruyter/Lucius \& Lucius via Zeitschrift für Soziologie, http://www.zfs-online.org/index.php/zfs/article/viewFile/3208/2745

Die materiellen Kräfteverhältnisse des Diskurskontextes sind ab den 1980er Jahren durch die zunehmende Bedeutung von Drittmitteln (vgl. Münch 2011) sowie durch Universitätsreformen charakterisiert, von denen die Bologna-Reform (vgl. Maeße 2010) den größten Einfluss auf den Bildungsdiskurs hat. Der Zwang zur Drittmitteleinwerbung steht erstens in Konkurrenz zur Lehrtätigkeit und gefährdet damit die gerade in den Geisteswissenschaften zentrale Einheit von Forschung und Lehre (Meier \& Schimank 2009; vgl. nur die Mahnungen in Frühwald 1991: 90; Nida-Rümelin 2005: 26), zweitens begünstigt er projektförmige gegenüber explorativer Einzelforschung (Torka 2009) und drittens übt er auf geisteswissenschaftliche Forschung den Druck aus, die eigene Relevanz offensiv zu thematisieren (Böhler \& Maasen 2011). Am besten gelingt dies offenbar einem kleinen Kreis immer gleicher Standorte: Die Deutsche Forschungsgemeinschaft gibt die Drittmittelförderung pro Professor/-in in den Sozial- und Geisteswissenschaften in vier Erhebungszeiträumen von 1991 bis 2010 an. Die für die jeweiligen Zeiträume ermittelten zehn Spitzenplätze sind von insgesamt nur 18 Standorten belegt. Mit Bielefeld, Konstanz, Stuttgart und Tübingen sind vier dieser Standorte mit den meisten Drittmitteln pro Professor/-in sogar über die gesamten 20 Jahre hinweg in der Spitzengruppe der zehn drittmittelstärksten Standorte vertreten (vgl. Tab. 1).

Tabelle 1 Standorte in den Sozial- und Geisteswissenschaften mit den jeweils insgesamt höchsten DFG-Bewilligungen pro Professor/-in, 1991-2010

\begin{tabular}{|l|l|l|l|l|}
\hline & $\mathbf{1 9 9 1 - 1 9 9 5}$ & $\mathbf{1 9 9 6 - 1 9 9 8}$ & $\mathbf{2 0 0 0 - 2 0 0 4}$ & $\mathbf{2 0 0 8 - 2 0 1 0}$ \\
\hline & 1. Bielefeld & 1. Konstanz & 1. Konstanz & 1. Konstanz \\
& 2. Stuttgart & 2. Bielefeld & 2. Tübingen & 2. FU Berlin \\
& 3. Freiburg & 3. Stuttgart & 3. Mannheim & 3. Heidelberg \\
Top 10 & 4. Düsseldorf & 4. Tübingen & 4. Bielefeld & 4. Münster \\
Stand- & 5. Konstanz & 5. Trier & 5. Freiburg & 5. Stuttgart \\
orte & 6. Siegen & 6. Bonn & 6. Heidelberg & 6. HU Berlin \\
& 7. Bayreuth & 7. Heidelberg & 7. FU Berlin & 7. Bielefeld \\
& 8. Bonn & 8. Freiburg & 8. Stuttgart & 8. Mannheim \\
& 9. Tübingen & 9. HU Berlin & 9. Jena & 9. Tübingen \\
& 10. Trier & 10. U München & 10. U München & 10. Bremen \\
\hline
\end{tabular}

(Deutsche Forschungsgemeinschaft 1997: 53; 2000: 94-95; 2006: 156-157; 2012: 115; eigene Berechnungen)

Auch der Einfluss der Bologna-Reform verdeutlicht sich im Zeitverlauf. Zwischen 2005 und 2012 steigt der Anteil der Bachelor- und Masterstudiengänge am gesamten Studienangebot in den Sprach- und Kulturwissenschaften von 13,6 auf 75,9 Prozent (Hochschulrektorenkonferenz 2012: 38). Innerhalb von sieben Jahren wird demnach ein großer Anteil der Lehrstruktur geis- 
These are proofs of the final publication that is available at de Gruyter/Lucius \& Lucius via Zeitschrift für Soziologie, http://www.zfs-online.org/index.php/zfs/article/viewFile/3208/2745

teswissenschaftlicher Fächer umgestellt. Es kann nicht überraschen, dass sich diese Kräfteverhältnisse im Bildungsdiskurs niederschlagen. Sie erscheinen dort erstens in Form von Klagen über die Bürokratisierung und Normierung der Bildungspraxis. Eine Verarbeitung des institutionellen Zwangs zur Drittmitteleinwerbung und der damit einhergehenden Symbolisierung von Relevanz kann zweitens in der Anrufung der Orientierungsfunktion von Bildung gesehen werden, während drittens die Zwecksetzung der Arbeitsmarktbefähigung eine diskursive Passung mit der auf diesen Aspekt abzielenden Bologna-Reform darstellt.

\section{Der Distinktionswert „freier“" und ,wissenschaftlicher" Bildung}

Bis hierher ermöglicht die Analyse der Zusammenhänge zwischen dem Bildungsdiskurs und seinen Kontexten eine Vorstellung davon, mit welcher Dynamik sich geisteswissenschaftliche Bildungsbegriffe und damit verbundene Selbstverständnisse im Zeitverlauf entwickeln und auf die materiellen und symbolischen Strukturen verweisen, in die sie eingebettet sind. Die Responsivität des Bildungsdiskurses für sozialen Wandel sollte jedoch nicht über seine Beharrungskraft hinwegtäuschen. Von einer Beharrungskraft des Diskurses kann gesprochen werden, insofern grundlegende inhaltliche Orientierungen von Bildungsbegriffen trotz des tief greifenden Wandels ihrer Produktions- und Rezeptionskontexte bestehen bleiben. Diese Stabilitäten in der Konstruktion von „Bildung“ verweisen auf die Eigenlogik diskursiver Formationsregeln gegenüber den Umwälzungen der sie umgebenden Diskurskontexte.

Zwei Topoi verfügen historisch über eine äußerst große Beharrungskraft. Erstens wird geisteswissenschaftlicher Bildung über 200 Jahre und alle Veränderungen des Feldkontextes hinweg „Freiheit“ zugeschrieben. Die Anrufung „freier“ Bildung bildet einen Konsens, der den gesamten Untersuchungszeitraum überspannt und jede Diskursfraktion integriert. Differenzen bestehen lediglich darin, wie selbstbewusst diese „Freiheit“ angesichts der jeweiligen Kontextbedingungen behauptet werden kann und wie hoch der Aufwand ist, mit dem sie gerechtfertigt werden muss. Während der Topos der „Freiheit“ demnach alle vier Phasen des Untersuchungszeitraums durchzieht, variiert sein Gegenüber im Zeitverlauf: Wird „freie“ Bildung in der ersten Phase durch unterschiedliche Grade der Distanz zum preußischen Staat bestimmt, dessen kulturnationales Programm die Bildung der Geisteswissenschaften überhaupt erst fundiert (vgl. Schleiermacher 1956: 263; Fichte 1910: 17), so definiert sie sich um die Wende zum 20. Jahrhundert in Abgrenzung zu den Praxisbezügen beruflicher Bildung, die nun in Reaktion auf gestiegene Studierendenzahlen wichtiger werden (vgl. Nietzsche 1954: 190-191; Scheler 1954: 32). In der dritten Phase von 1945 bis zum Ende der 1970er Jahre stellen Lehrpläne und Studi- 
These are proofs of the final publication that is available at de Gruyter/Lucius \& Lucius via Zeitschrift für Soziologie, http://www.zfs-online.org/index.php/zfs/article/viewFile/3208/2745

enordnungen, die Bildung in der durch die Bildungsexpansion geöffneten Universität normieren und strukturieren, das Gegenüber der „Freiheit“ dar (vgl. Jaspers 1986: 235; Ritter 1974: 109). In der letzten Phase von der Mitte der 1980er Jahre bis in die Gegenwart aktualisiert sich die Behauptung der „Freiheit“ gegenüber politisch oktroyierten Bildungsreformen und der Marktförmigkeit von Bildung (vgl. Arnswald 2005: 147; Brandt 2011: 169-170). In jeder Phase bleibt die Feststellung, dass Bildung nicht frei, sondern zum Beispiel eine von den Geisteswissenschaften erbrachte Leistung für die Gesellschaft ist, völlig unsagbar. In den jeweiligen Anderen, an denen sich die Behauptung der Freiheit konturiert, scheint jedoch auf, welchen gesellschaftlichen Einflüssen die Geisteswissenschaften in Preußen, dem Kaiserreich, der Weimarer, der Bonner und der Berliner Republik unterliegen.

Der Topos der „Freiheit“ ist zweitens begleitet vom Topos der „Wissenschaftlichkeit“" von Bildung. Auch diese Ausdeutung erstreckt sich über alle vier Phasen des Untersuchungszeitraums und über alle Fraktionen des Diskurses. Ihre konkrete Ausdeutung aktualisiert sich dabei im Zeitverlauf mit Bezug auf ein historisch variables Gegenüber. Informiert durch den Deutschen Idealismus meint ,wissenschaftliche“ Bildung in der ersten Phase die Vermittlung universalistischer Prinzipien, die die Einheit des Wissens repräsentieren (vgl. Krug 1805: 36; Steffens 1910: 217). In der zweiten Phase konturiert sich dieser Topos im Zusammenhang mit einer Forschungspraxis, in die wissenschaftliche (Aus-)Bildung einführen soll. Das Andere, auf das ,wissenschaftliche“ Bildung jetzt Bezug nimmt, ist ein von den Naturwissenschaften beeinflusstes, an empirischen Gegenständen und Methoden orientiertes Forschungsklima (vgl. Zeller 1879: 19; Wilamowitz-Moellendorff o.J.: 291, 318). In der dritten Phase definiert sich „Wissenschaftlichkeit“ über den Zusatz, dass wissenschaftliche Bildung auch ein wichtiges Element der Ausbildung für akademische Berufe sei (vgl. Litt 1952: 16-17; Habermas 1969c: 101). Schließlich wird „wissenschaftliche“ Bildung in der letzten Phase gegenüber Tendenzen der Verschulung und der Spezialisierung positioniert und aktualisiert sich in Anrufungen der Einheit von Forschung und Lehre (vgl. Frühwald 1991: 74, 76; Liessmann 2006: 107, 113). Die Feststellung, dass Bildung, ,wissenschaftlich“ sei, bleibt also über mehr als 200 Jahre hinweg unstrittig und stellt die zweite Konstante im Bildungsdiskurs dar. Im Rahmen einer Positionierungsarbeit wird der Topos der „Wissenschaftlichkeit“ an den jeweiligen Demarkationslinien zwischen den Geisteswissenschaften und ihrem gesellschaftlichen Außen in Stellung gebracht und zu diesem Gegenüber in jeder Phase neu in Beziehung gesetzt.

Die von der Analyse freigelegten Kontinuitäten geisteswissenschaftlicher Bildungsbegriffe ergänzen die Kartierung der Disziplinengruppe. Ungeachtet des sozialen Wandels und neben 
These are proofs of the final publication that is available at de Gruyter/Lucius \& Lucius via Zeitschrift für Soziologie, http://www.zfs-online.org/index.php/zfs/article/viewFile/3208/2745

den Um- und Neudefinitionen einzelner inhaltlicher Dimensionen von „Bildung“ bleiben „Freiheit“" und „Wissenschaftlichkeit“ zentrale Ausprägungen im Bildungsdiskurs. Ihre Ambivalenzen werden jeweils bei Abgrenzungen gegen ein historisch variables Anderes aktualisiert und konturiert. In zweierlei Hinsicht spielen die beiden Topoi eine wichtige Rolle für die Konstruktion geisteswissenschaftlicher Selbstverständnisse (vgl. Gieryn 1983; Abbott 1995).

Sie verfügen erstens über das integrative Potenzial, die Disziplinengruppe trotz aller Konfliktlinien ideologisch zu einen. „Wissenschaftlichkeit“ markiert nicht nur geisteswissenschaftliche Bildung, sondern die Geisteswissenschaften insgesamt als dezidiert akademisches Unterfangen, das sich seiner Differenz zu intellektuellen Betätigungen in der Kunst, im Feuilleton oder der Literatur auch intern vergewissern muss. Diese Notwendigkeit ergibt sich aus den Besonderheiten des geisteswissenschaftlichen Feldes: Intellektuelle Prominenz stellt hier eine Art weltliches Kapital dar, gegenüber dem ein explizit wissenschaftlicher Status markiert werden muss (Bourdieu 1992: 133). In diesem Sinne ist die Beharrung auf ,wissenschaftlicher“ Bildung eine integrative Strategie, mit der sich die Geisteswissenschaften als dezidiert „wissenschaftliche" epistemische Kultur versichern, die sich von benachbarten intellektuellen Betätigungen unterscheidet. Ganz ähnlich verweist das Beharren auf „Freiheit“" auf die von allen Akteuren des geisteswissenschaftlichen Feldes geteilte Illusio einer autonomen, reinen Wissenschaft (Bourdieu 1992), auf die unhintergehbare Überzeugung also, dass die Auseinandersetzungen und Kämpfe einer Logik folgen, die nur den Geisteswissenschaften zu eigen ist.

Zweitens haben die Topoi der „Wissenschaftlichkeit“ und „Freiheit“ eine zentrale Bedeutung für die Konstruktion geisteswissenschaftlicher Selbstverständnisse, weil sie als Abgrenzung gegenüber einem gesellschaftlichen und akademischen Außen fungieren. In diesem Sinne liegt die konstitutive Bedeutung von „Wissenschaftlichkeit“ in der Relation des geisteswissenschaftlichen Feldes zu anderen Feldern und ihren Diskursen: Zunächst verspricht „Wissenschaftlichkeit" hohe symbolische Profite im Akademisierungsprozess, mit dem sich das Feld an der Wende zum 19. Jahrhundert von staatlichen und religiösen Zugriffen emanzipiert. Seit dem letzten Drittel des 19. Jahrhunderts werden die Geisteswissenschaften dann von den Natur- und den Sozialwissenschaften unter Druck gesetzt und müssen sich mit einem eigenständigen wissenschaftlichen Status abgrenzen. In dieser symbolisch schon immer prekären Lage ist es für den Erhalt der Eigenlogik des geisteswissenschaftlichen Feldes existenziell, nicht ausschließlich fremde Wissenschaftsverständnisse zu übernehmen, sondern seinerseits auf einer genuinen „Wissenschaftlichkeit“ zu beharren. Indem die Berufung auf „Freiheit“ einen von äußeren Relevanzforderungen autonomen identitären Kern symbolisiert, fungiert auch sie als Grenzarbeit gegenüber gesellschaftlichen Einflussnahmen. Gerade im Lichte der Vereinnahmungen vom 
These are proofs of the final publication that is available at de Gruyter/Lucius \& Lucius via Zeitschrift für Soziologie, http://www.zfs-online.org/index.php/zfs/article/viewFile/3208/2745

absolutistischen Preußen bis zur Berliner Republik ist es entscheidend, „frei“ zu sein und „reine“ Wissenschaft und Bildung zu betreiben - nicht zuletzt gründet auf dieser Zuschreibung überhaupt erst das gesellschaftliche Interesse an (geistes-)wissenschaftlicher Expertise. Hierin zeigt sich das ideologische Erbe Kants (2005: 27), der die philosophische Fakultät bekanntlich gerade deshalb zur oberen Fakultät erklärt, weil er in ihr „das Vermögen, nach der Autonomie, d.i. frei $[\ldots]$ zu urteilen“ sieht und sie deshalb ,als frei und nur unter Gesetzgebung der Vernunft, nicht der Regierung stehend“ konzipiert.

\section{Fazit}

Mit dem vorliegenden Beitrag soll der Fokussierung der Wissenschaftssoziologie auf Naturund Technikwissenschaften die Kartierung eines vernachlässigten Gegenstandsbereichs entgegengesetzt werden. Welche (wissenschafts-)soziologischen Einsichten ermöglicht eine historisch informierte Wissenschaftssoziologie der Geisteswissenschaften also? Die Genealogie der „Bildung“ der Geisteswissenschaften konnte sowohl Beharrungskräfte des geisteswissenschaftlichen Selbstverständnisses als auch eine Responsivität für sozialen Wandel rekonstruieren. Bei dieser Gleichzeitigkeit von diskursiver Sensibilität und Beharrungsvermögen handelt es sich nur auf den ersten Blick um widersprüchliche diskursive Dynamiken. Ihre Kombination wird ermöglicht durch die produktive Verknüpfung legitimer, über lange Zeiträume hinweg gültiger Bildungsideale mit einer durch konkrete Kräfteverhältnisse geprägten und tendenziell illegitimen Bildungsrealität. Die Kombination dieser Motive stellt einen diskursiven Mechanismus dar, der dem Beharren auf traditionellen Topoi fortlaufend Legitimität verleiht, weil gleichzeitig Veränderungen der Bildungsrealität diskursiv verarbeitet und kompensiert werden können. Erst in der Gegenüberstellung mit den Gefahren und Dysfunktionalitäten der geisteswissenschaftlichen Realität lassen sich Idealvorstellungen über einen langen Zeitraum hinweg als historische Projektionsfläche aufrechterhalten und für die jeweils aktuelle Situation aktualisieren.

Durch die vorgenommene Kartierung können die auf die gegenwärtige Situation der Geisteswissenschaften zielenden Fragen vom Beginn dieses Artikels auf aktuelle Diskussionen in der Wissenschaftssoziologie bezogen werden: Erstens ist die Krisenaffinität der Geisteswissenschaften aus diachroner Perspektive kein völlig neues Phänomen. Spätestens im letzten Drittel des 19. Jahrhunderts fordern Industrialisierung und Modernisierung geisteswissenschaftliche Orientierungen und die damit verbundenen Bildungsbegriffe heraus. Die aktuell besonders ausgeprägte Tendenz zu Krisendiagnosen wird verständlicher, wenn berücksichtigt wird, dass eine wissenschafts- und hochschulpolitische Realität, die durch Folgen der Bildungsexpansion und den politisch forcierten Wettbewerb um Ressourcen gekennzeichnet ist (vgl. Slaughter \& Leslie 
These are proofs of the final publication that is available at de Gruyter/Lucius \& Lucius via Zeitschrift für Soziologie, http://www.zfs-online.org/index.php/zfs/article/viewFile/3208/2745

1999; Münch 2011), innerhalb einer beharrlichen Orientierung an „Freiheit“ und „Wissenschaftlichkeit“" verarbeitet werden muss. Gegenüber diesen Topoi markieren die aktuellen Behauptungen einer verschulten und bürokratisch normierten Bildung krisenhafte Auswüchse.

Zweitens ist deutlich geworden, dass eine Einheit der „Geisteswissenschaften“ schon lange einer sich differenzierenden Fachkommunikation gegenübersteht. Wie gezeigt wurde, muss der Bildungsdiskurs bereits im späten 19. Jahrhundert als Ort der Konstruktion eines ganzheitlichen geisteswissenschaftlichen Selbstverständnisses immer spezialisiertere Forschungsbereiche überbrücken. Es sind gerade holistische Selbstverständigungen wie der Bildungsdiskurs, die die Geisteswissenschaften gegenüber Tendenzen der disziplinären Spezialisierung und Differenzierung (vgl. Abbott 2001; Becher \& Trowler 2001; Jacobs 2013) immer wieder zu einer epistemischen Kultur vereinen und als einheitliche Gruppe markieren. Für diese nach innen und nach außen gerichtete Positionierung spielen die Topoi einer „freien“ und „wissenschaftlichen“ Bildung eine besondere Rolle.

Drittens hat die historisch informierte Analyse verdeutlicht, dass die Geisteswissenschaften und ihre Bildung schon immer in gesellschaftliche Verwertungszusammenhänge eingebunden und responsiv für deren Relevanzforderungen waren. Bereits der preußische Staat hat die charakterbildende Funktion geisteswissenschaftlicher Bildung nur anerkannt, weil sie seiner Räson dienlich erschien. Auch die in den folgenden Phasen vorgenommenen Funktionszuschreibungen hatten stets die Verwertbarkeit von Bildung außerhalb akademischer Zusammenhänge im Blick. Die Einbindung der Geisteswissenschaften in nationalstaatliche Projekte (vgl. Berger 1997; Bontempelli 2004; Gengnagel \& Hamann 2014) war bisher mitnichten ein Hindernis für die Symbolisierung von „Freiheit“ und „Wissenschaftlichkeit“. Offensichtlich lassen sich diese Topoi jedoch nicht ohne Weiteres mit aktuellen Relevanzforderungen und Leistungsbewertungen verbinden (siehe nur Nederhof 2006; Lack \& Markschies 2008). Hierin kann eine Erklärung für die Probleme mit ,impact“- und drittmittelorientierten Instrumenten der Hochschulsteuerung gesehen werden. Gegenwärtige Behauptungen der Bedeutung von Bildung für den Arbeitsmarkt sowie ihrer individuellen und gesellschaftlichen Orientierungsfunktion sind bislang offenbar nur bedingt erfolgreiche Versuche einer Inwertsetzung geisteswissenschaftlicher Leistungen. Zum Verständnis dieser und anderer Themenkomplexe kann eine vertiefte wissenschaftssoziologische Erforschung der Geisteswissenschaften beitragen.

\section{Literatur}

Abbott, A., 1995: Things of Boundaries. Social Research 62: 857-882.

Abbott, A., 2001: Chaos of Disciplines. Chicago: University of Chicago Press. 
These are proofs of the final publication that is available at de Gruyter/Lucius \& Lucius via Zeitschrift für Soziologie, http://www.zfs-online.org/index.php/zfs/article/viewFile/3208/2745

Adorno, T.W., 1986 (zuerst 1959): Zur Demokratisierung der deutschen Universitäten. S. $332-338$ in: R. Tiedemann (Hrsg.), Theodor W. Adorno. Gesammelte Schriften, Bd. 20.1: Vermischte Schriften 1. Frankfurt/M.: Suhrkamp.

Adorno, T.W., 2006 (zuerst 1959): Theorie der Halbbildung. Frankfurt/M.: Suhrkamp.

Adorno, T.W., R. Dahrendorf, H. Pilot, H. Albert, J. Habermas \& K.R. Popper (Hrsg.), 1972: Der Positivismusstreit in der deutschen Soziologie. Darmstadt, Neuwied: Luchterhand.

Angermüller, J., 2007: Nach dem Strukturalismus. Theoriediskurs und intellektuelles Feld in Frankreich. Bielefeld: transcript.

Angermüller, J., 2010: Beyond Excellence - An Essay on the Social Organization of the Social Sciences and Humanities. Sociologica 2010: 1-16.

Anrich, E., 1960: Die Idee der deutschen Universität und die Reform der deutschen Universitäten. Darmstadt: Wissenschaftliche Buchgesellschaft.

Arnswald, U., 2005: Die Geisteswissenschaften - unterschätzte Transmissionsriemen des gesellschaftlichen Wandels und der Innovation. S. 111-162 in: U. Arnswald (Hrsg.), Die Zukunft der Geisteswissenschaften. Heidelberg: Manutius Verlag.

Baumgarten, M., 1997: Professoren und Universitäten im 19. Jahrhundert: zur Sozialgeschichte deutscher Geistesund Naturwissenschaftler. Göttingen: Vandenhoeck \& Ruprecht.

Baumgartner, H.M. \& H.-M. Sass, 1979: Philosophie in Deutschland 1945-1975. Standpunkte - Entwicklungen Literatur. Königstein/Ts.: Anton Hain.

Becher, T. \& P. Trowler, 2001: Academic Tribes and Territories: Intellectual Enquiry and the Cultures of Disciplines. Philadelphia: Open University Press.

Beer, B. \& M. Koenig, 2009: Grenzziehungen im System wissenschaftlicher Disziplinen - der Fall der Kulturwissenschaft(en). Sociologia Internationalis 47: 3-38.

Behrens, J., L. Fischer, K.-H. Minks \& L. Rösler, 2010: Die internationale Positionierung der Geisteswissenschaften in Deutschland. Eine empirische Untersuchung. Hannover: HIS.

Beiser, F.C., 2008: Historicism and neo-Kantianism. Studies in History and Philosophy of Science 39: 554-564.

Ben-David, J., 1971: The Scientist's Role in Society. A Comparative Study. Englewood Cliffs, New Jersey: Prentice Hall.

Benneworth, P. \& B.W. Jongbloed, 2010: Who Matters to Universities? A Stakeholder Perspective on Humanities, Arts and Social Sciences Valorisation. Higher Education 59: 567-588.

Berger, S., 1997: The Search for Normality. National Identity and Historical Consciousness in Germany since 1800. New York, Oxford: Berghahn Books.

Bod, R., J. Maat \& T. Weststeijn (Hrsg.), 2014: The Making of the Humanities III. The Modern Humanities. Amsterdam: Amsterdam University Press.

Böhler, F. \& S. Maasen, 2011: Die Transformation der Geisteswissenschaften 1990-2007. S. 63-89 in: B. Hölscher \& J. Suchanek (Hrsg.), Wissenschaft und Hochschulbildung im Kontext von Wirtschaft und Medien. Wiesbaden: VS.

Bollenbeck, G., 1994: Bildung und Kultur. Glanz und Elend eines deutschen Deutungsmusters. Frankfurt/M.: Insel Verlag.

Bontempelli, P.C., 2004: Knowledge, Power, and Discipline: German Studies and National Identity. Minneapolis: University of Minnesota Press.

Bourdieu, P., 1975: The Specificity of the Scientific Field and the Social Conditions of the Progress of Reason. Social Science Information 14: 19-47.

Bourdieu, P., 1992: Homo academicus. Frankfurt/M.: Suhrkamp.

Brandt, H.-H., 2001: Studierende im Humboldt'schen Modell des 19. Jahrhunderts. S. 131-150 in: R.C. Schwinges (Hrsg.), Humboldt International. Der Export des deutschen Universitätsmodells im 19. und 20. Jahrhundert. Basel: Schwabe \& Co.

Brandt, R., 2011: Wozu noch Universitäten? Hamburg: Felix Meiner.

Brenner, P.J., 1997: Die Grenzen des Geistes. Zur Infrastruktur geisteswissenschaftlicher Arbeit. S. 45-90 in: H. Reinalter \& R. Benedikter (Hrsg.), Geisteswissenschaften wozu? Studien zur Situation der Geisteswissenschaften. Thaur: Druck- und Verlagshaus Thaur.

Brownley, M.W., 2012: Academic Specialization and Contemporary University Humanities Centers. Arts and Humanities in Higher Education 11: 224-237.

Bundesassistentenkonferenz (Hrsg.), 1971: Texte zur Studienreform, Bd. 1: Kritische Germanistik: eine hermeneutische und materialistische Wissenschaft. Bonn: BAK.

Busch, A., 1977: Die Geschichte des Privatdozenten. New York: Arno Press.

Camic, C., N. Gross \& M. Lamont (Hrsg.), 2011: Social Knowledge in the Making. Chicago: University of Chicago Press.

Charle, C., 2004: Grundlagen. S. 43-80 in: W. Rüegg (Hrsg.), Geschichte der Universität in Europa. Bd. III: Vom 19. Jahrhundert zum zweiten Weltkrieg (1800-1945). München: C.H. Beck.

Chomsky, N., 1973: Strukturen der Syntax. The Hague: Mouton. 
These are proofs of the final publication that is available at de Gruyter/Lucius \& Lucius via Zeitschrift für Soziologie, http://www.zfs-online.org/index.php/zfs/article/viewFile/3208/2745

Collins, R., 2000: The Sociology of Philosophies: A Global Theory of Intellectual Change. Harvard: Harvard University Press.

Dayé, C., 2014: Visions of a Field: Recent Developments in Studies of Social Sciences and Humanities. Science, Technology, \& Human Values 39: 877-891.

Deutsche Forschungsgemeinschaft, 1997: Bewilligungen nach Hochschulen. Bewilligungsvolumen 1991 bis 1995, Anzahl kooperativer Projekte im Jahr 1996. Bonn: DFG.

Deutsche Forschungsgemeinschaft, 2000: DFG-Bewilligungen an Hochschulen und außeruniversitäre Forschungseinrichtungen 1996 bis 1998. Bonn: DFG.

Deutsche Forschungsgemeinschaft, 2006: Förder-Ranking 2006. Regionen - Institutionen - Netzwerke. Bonn: DFG.

Deutsche Forschungsgemeinschaft, 2012: Förderatlas 2012. Kennzahlen zur öffentlich finanzierten Forschung in Deutschland. Bonn: DFG.

Diaz-Bone, R., 2002: Kulturwelt, Diskurs und Lebensstil. Eine diskurstheoretische Erweiterung der bourdieuschen Distinktionstheorie. Opladen: Leske + Budrich.

Diaz-Bone, R., 2005: Die »interpretative Analytik« als rekonstruktiv-strukturalistische Methodologie. S. 179-197 in: R. Keller, A. Hirseland, W. Schneider \& W. Viehöver (Hrsg.), Die diskursive Konstruktion von Wirklichkeit. Konstanz: UVK.

Dilthey, W., 1958 (zuerst 1910): Der Aufbau der geschichtlichen Welt in den Geisteswissenschaften. S. in: B. Groethuysen (Hrsg.), Wilhelm Dilthey. Gesammelte Schriften, Bd. VII. Stuttgart: B.G. Teubner.

Dilthey, W., 1959 (zuerst 1883): Einleitung in die Geisteswissenschaften. Versuch einer Grundlegung für das Studium der Gesellschaft und der Geschichte. S. in: B. Groethuysen (Hrsg.), Wilhelm Dilthey. Gesammelte Schriften, Bd. I. Stuttgart: B.G. Teubner.

Donoghue, F., 2008: The Last Professors. The Corporate University and the Fate of the Humanities. New York: Fordham University Press.

Dreyfus, H.L. \& P. Rabinow, 1987: Michel Foucault. Jenseits von Strukturalismus und Hermeneutik. Frankfurt/M.: Athenäum.

Enders, J., 1998: Berufsbild der Hochschullehrer. S. 55-78 in: U. Teichler, H.-D. Daniel \& J. Enders (Hrsg.), Brennpunkt Hochschule. Neuere Analysen zu Hochschule, Beruf und Gesellschaft. Frankfurt/M., New York: Campus.

Enders, J. \& U. Schimank, 2001: Faule Professoren und vergreiste Nachwuchswissenschaftler. Einschätzungen und Wirklichkeit. S. 159-178 in: E. Stölting \& U. Schimank (Hrsg.), Die Krise der Universitäten. Leviathan Sonderheft 20. Opladen: Westdeutscher Verlag.

Fabian, B., 1996: Die Geisteswissenschaften - nach ihrer »Krise«. S. 13-39 in: B. Fabian (Hrsg.), Zukunftsaspekte der Geisteswissenschaften. Vier Vorträge. Hildesheim: Olms-Weidmann.

Fichte, J.G., 1845 (zuerst 1794): Einige Vorlesungen über die Bestimmung des Gelehrten. S. 291-346 in: I.H. Fichte (Hrsg.), Johann Gottlieb Fichtes sämmtliche Werke, Bd. 6. Berlin: Verlag von Veit und Comp.

Fichte, J.G., 1910 (zuerst 1807): Deducirter Plan einer zu Berlin zu errichtenden höhern Lehranstalt. S. 1-104 in: E. Spranger (Hrsg.), Fichte, Schleiermacher, Steffens über das Wesen der Universität. Leipzig: Verlag der Dürr'schen Buchhandlung.

Fichte, J.G., 1997 ([1794]): Über den Begriff der Wissenschaftslehre. S. in: W.G. Jacobs (Hrsg.), Werke, Band 1. Frankfurt/M.: Deutscher Klassiker-Verlag.

Foucault, M., 1981: Archäologie des Wissens. Frankfurt/M.: Suhrkamp.

Foucault, M., 1996 (zuerst 1983): Diskurs und Wahrheit. Berkeley-Vorlesungen 1983. Berlin: Merve.

Friese, S., 2012: Qualitative Data Analysis with ATLAS.ti. London et al.: SAGE.

Frühwald, W., 1991: Humanistische und naturwissenschaftlich-technische Bildung: die Erfahrung des 19. Jahrhunderts. S. 73-111 in: W. Frühwald, H.R. Jauß, R. Koselleck, J. Mittelstraß \& B. Steinwachs (Hrsg.), Geisteswissenschaften heute. Frankfurt/M.: Suhrkamp.

Gauger, J.-D. \& G. Rüther (Hrsg.), 2007: Warum die Geisteswissenschaften Zukunft haben! Ein Beitrag zum Wissenschaftsjahr 2007. Freiburg: Herder.

Gengnagel, V. \& J. Hamann, 2014: The Making and Persisting of Modern German Humanities. Balancing Acts between Autonomy and Social Relevance. S. 641-654 in: R. Bod, J. Maat \& T. Weststeijn (Hrsg.), The Making of the Humanities III. The Modern Humanities. Amsterdam: Amsterdam University Press.

Gethmann, C.F., D. Langewiesche, J. Mittelstraß, D. Simon \& G. Stock, 2005: Manifest Geisteswissenschaft. Berlin: Berlin-Brandenburgische Akademie der Wissenschaften.

Gieryn, T.F., 1983: Boundary-Work and the Demarcation of Science from non-Science: Strains and Interests in Professional Ideologies of Scientists. American Sociological Review 48: 781-795.

Groh, D., 1973: Kritische Geschichtswissenschaft in emanzipatorischer Absicht. Überlegungen zur Geschichtswissenschaft als Sozialwissenschaft. Stuttgart: Kohlhammer.

Guetzkow, J., M. Lamont \& G. Mallard, 2004: What is Originality in the Humanities and the Social Sciences? American Sociological Review 69: 190-212. 
These are proofs of the final publication that is available at de Gruyter/Lucius \& Lucius via Zeitschrift für Soziologie, http://www.zfs-online.org/index.php/zfs/article/viewFile/3208/2745

Guillory, J., 1993: Cultural Capital. The Problem of Literary Canon Formation. Chicago, London: University of Chicago Press.

Habermas, J., 1969a (zuerst 1968/1969): Für ein neues Konzept der Hochschulverfassung. S. 202-243 in: J. Habermas (Hrsg.), Protestbewegung und Hochschulreform. Frankfurt/M.: Suhrkamp.

Habermas, J., 1969b (zuerst 1967): Universität in der Demokratie - Demokratisierung der Universität. S. 108-133 in: J. Habermas (Hrsg.), Studentenprotest und Hochschulreform. Frankfurt/M.: Suhrkamp.

Habermas, J., 1969c (zuerst 1966): Zwangsjacke für die Studienreform. Die befristete Immatrikulation und der falsche Pragmatismus des Wissenschaftsrats. S. 92-107 in: J. Habermas (Hrsg.), Protestbewegung und Hochschulreform. Frankfurt/M.: Suhrkamp.

Hall, S., 1990: The Emergence of Cultural Studies and the Crisis of the Humanities. October 1990: 11-23.

Hamann, J., 2011: 'Bildung' in German human sciences: The discursive transformation of a concept. History of the Human Sciences 24: 48-72.

Hamann, J., 2014: Die Bildung der Geisteswissenschaften. Zur Genese einer sozialen Konstruktion zwischen Diskurs und Feld. Konstanz: UVK.

Hegel, G.W.F., 1907 (zuerst 1807): Phänomenologie des Geistes. Leipzig: Verlag der Dürr'schen Buchhandlung.

Heidbrink, L. \& H. Welzer (Hrsg.), 2007: Das Ende der Bescheidenheit. Zur Verbesserung der Geistes- und Kulturwissenschaften. München: Beck.

Heimpel, H., 1956: Probleme und Problematik der Hochschulreform. Göttingen: Schwartz.

Heintz, B., 1993: Wissenschaft im Kontext. Neue Entwicklungstendenzen der Wissenschaftssoziologie. Kölner Zeitschrift für Soziologie und Sozialpsychologie 45: 528-552.

Herbert, U., 2009: Geisteswissenschaftliche Standards in Forschung und Lehre. S. 31-42 in: K.H. Hempfer \& P. Anthony (Hrsg.), Zur Situation der Geisteswissenschaften in Forschung und Lehre. Eine Bestandsaufnahme aus der universitären Praxis. Stuttgart: Franz Steiner.

Herrmann, H.P., 1989: Abschaffung der Geisteswissenschaften? Standortbestimmung im aktuellen Streit zwischen Politik und Hochschulen. S. 50-65 in: J. Förster, E. Neuland \& G. Rupp (Hrsg.), Wozu noch Germanistik? Wissenschaft - Beruf - Kulturelle Praxis. Stuttgart: J.B. Metzlersche Verlagsbuchhandlung.

Hochschulrektorenkonferenz, 1992: Mehr Autonomie für die Hochschulen. Zur Deregulierung im Hochschulrecht des Bundes und der Länder. Bonn: Hochschulrektorenkonferenz.

Hochschulrektorenkonferenz, 2012: Statistische Daten zu Studienangeboten an Hochschulen in Deutschland. Studiengänge, Studierende, Absolventen, Wintersemester 2012/2013. Statistiken zur Hochschulpolitik November 2012. Bonn: HRK.

Hollinger, D.A. (Hrsg.), 2006: The Humanities and the Dynamics of Inclusion since World War II. Baltimore, MD: John Hopkins University Press.

Humboldt, W.v., 1956 (zuerst 1810): Über die innere und äußere Organisation der höheren wissenschaftlichen Anstalten in Berlin. S. 375-386 in: E. Anrich (Hrsg.), Die Idee der deutschen Universität. Die fünf Grundschriften aus der Zeit ihrer Neubegründung durch klassischen Idealismus und romantischen Realismus. Darmstadt: Wissenschaftliche Buchgesellschaft.

Humboldt, W.v., 1960 (zuerst 1793): Theorie der Bildung des Menschen. S. 234-240 in: A. Flitner \& K. Giel (Hrsg.), Wilhelm von Humboldt. Werke in fünf Bänden, Bd. I. Darmstadt: Wissenschaftliche Buchgesellschaft.

Humboldt, W.v., 1964 (zuerst 1809): Bericht der Sektion des Kultus und Unterrichts an den König, Dezember 1809. S. 210-238 in: A. Flitner \& K. Giel (Hrsg.), Wilhelm von Humboldt. Werke in fünf Bänden, Bd. IV. Darmstadt: Wissenschaftliche Buchgesellschaft.

Jacobs, J.A., 2013: In Defense of Disciplines: Interdisciplinarity and Specialization in the Research University. Chicago: University of Chicago Press.

Jaeger, F. \& J. Rüsen, 1992: Geschichte des Historismus. München: C.H. Beck.

Jaeger, W., 1960 (zuerst 1923): Stellung und Aufgaben der Universität in der Gegenwart. S. 68-86 in: W. Jaeger (Hrsg.), Humanistische Reden und Vorträge. Berlin: De Gruyter \& Co.

Jaspers, K., 1946: Die Idee der Universität. Berlin: Springer.

Jaspers, K., 1986 (zuerst 1945/46): Vom lebendigen Geist der Universität. S. 215-241 in: K. Jaspers (Hrsg.), Erneuerung der Universität. Reden und Schriften. Heidelberg: Verlag Lambert Schneider.

Jauß, H.R., 1967: Literaturgeschichte als Provokation der Literaturwissenschaft. Konstanz: UVK.

Jay, P., 2014: The Humanities »Crisis« and the Future of Literary Studies. Houndmills, Basingstoke: Palgrave Macmillan.

Kagan, J., 2009: The Three Cultures: Natural Sciences, Social Sciences, and the Humanities in the 21st Century. Cambridge: Cambridge University Press.

Kaldewey, D., 2013: Wahrheit und Nützlichkeit. Selbstbeschreibungen der Wissenschaft zwischen Autonomie und gesellschaftlicher Relevanz. Bielefeld: transcript.

Kant, I., 2005 (zuerst 1798): Der Streit der Fakultäten. Hamburg: Felix Meiner.

Karabel, J., 2006: The Chosen. The Hidden History of Admission and Exclusion at Harvard, Yale, and Princeton. Boston, New York: Mariner Books. 
These are proofs of the final publication that is available at de Gruyter/Lucius \& Lucius via Zeitschrift für Soziologie, http://www.zfs-online.org/index.php/zfs/article/viewFile/3208/2745

Kath, G., 1977: Das soziale Bild der Studentenschaft in der Bundesrepublik Deutschland. Frankfurt/M.: k.A.

Kauppi, N., 1996: French Intellectual Nobility. Institutional and Symbolic Transformations in the Post-Sartrian Era. Albany: State University of New York Press.

Keller, R., 2005: Wissenssoziologische Diskursanalyse als interpretative Analytik. S. $49-75$ in: R. Keller, A. Hirseland, W. Schneider \& W. Viehöver (Hrsg.), Die diskursive Konstruktion von Wirklichkeit. Zum Verhältnis von Wissenssoziologie und Diskursforschung. Konstanz: UVK.

Keller, R., 2008: Wissenssoziologische Diskursanalyse. Grundlegung eines Forschungsprogramms. Wiesbaden: VS.

Kellermann, P., 2006: Von Sorbonne nach Bologna und darüber hinaus. Zur Ideologie derzeitiger Hochschulpolitik. Soziologie 35: 56-69.

Kernan, A. (Hrsg.), 1997: What's Happened to the Humanities? Princeton: Princeton University Press.

Klinge, M., 2004: Die Universitätslehrer. S. 113-143 in: W. Rüegg (Hrsg.), Geschichte der Universität in Europa. Bd. III: Vom 19. Jahrhundert zum Zweiten Weltkrieg (1800-1945). München: C.H. Beck.

Kneer, G. \& S. Moebius, 2010: Vorwort. S. 7-13 in: G. Kneer \& S. Moebius (Hrsg.), Soziologische Kontroversen. Beiträge zu einer Geschichte der Wissenschaft vom Sozialen. Frankfurt/M.: Suhrkamp.

Krebs, R., I. Siouti, U. Apitzsch \& S. Wenk, 2005: Disciplinary Barriers between the Social Sciences and Humanities: National Report on Germany. Retrieved November 2005: o.S.

Krug, W.T., 1805: Versuch einer neuen Eintheilung der Wissenschaften zur Begründung einer besseren Organisazion für die höheren gelehrten Bildungsanstalten. Züllichau, Freystadt: Darnmann.

Lack, E. \& C. Markschies (Hrsg.), 2008: What the Hell is Quality? Qualitätsstandards in den Geisteswissenschaften. Frankfurt/M., New York: Campus.

Lakoff, G., 1971: Linguistik und natürliche Logik. Frankfurt/M.: Athenäum.

Lamont, M., 2009: How Professors Think. Inside the Curious World of Academic Judgement. Cambridge, MA: Harvard University Press.

Lepenies, W., 1985: Die drei Kulturen: Soziologie zwischen Literatur und Wissenschaft. München: Hanser.

Liessmann, K.P., 2006: Theorie der Unbildung. Die Irrtümer der Wissensgesellschaft. Wien: Paul Zsolnay Verlag.

Litt, T., 1952: Der Bildungsauftrag der deutschen Hochschule. Vortrag, gehalten bei der Tagung des Hochschulverbandes in Marburg am 2. Mai 1952. Göttingen: Otto Schwartz \& Co.

Lundgreen, P., 1983: Differentiation in German Higher Education. S. 149-179 in: K.H. Jarausch (Hrsg.), The Transformation of Higher Learning. 1860-1930. Expansion, Diversification, Social Opening and Professionalization in England, Germany, Russia and the United States. Stuttgart: Ernst Klett.

Lundgreen, P., 2000: Bildung und Bürgertum. S. 173-194 in: P. Lundgreen (Hrsg.), Sozial- und Kulturgeschichte des Bürgertums. Göttingen: Vandenhoeck \& Ruprecht.

Maeße, J., 2010: Die vielen Stimmen des Bologna-Prozesses. Bielefeld: transcript.

Maeße, J., 2013: Das Feld und der Diskurs der Ökonomie. S. 241-275 in: J. Maeße (Hrsg.), Ökonomie, Diskurs, Regierung. Interdisziplinäre Perspektiven. Wiesbaden: VS.

Marquard, O., 1986: Über die Unvermeidlichkeit der Geisteswissenschaften. S. 98-116 in: O. Marquard (Hrsg.), Apologie des Zufälligen. Philosophische Studien. Stuttgart: Reclam.

Meier, F. \& U. Schimank, 2009: Matthäus schlägt Humboldt? New Public Management und die Einheit von Forschung und Lehre. Beiträge zur Hochschulforschung 31: 42-61.

Mittelstraß, J., 2002: Die Einheit der Bildung. Über die Rolle der Geisteswissenschaft in der modernen Welt. S. 71-81 in: K. Gloy (Hrsg.), Im Spannungsfeld zweier Kulturen. Eine Auseinandersetzung zwischen Geistesund Naturwissenschaft, Kunst und Technik. Würzburg: Königshausen \& Neumann.

Mommsen, W.J., 1971: Die Geschichtswissenschaft jenseits des Historismus. Düsseldorf: Droste.

Münch, R., 2007: Die akademische Elite. Zur sozialen Konstruktion wissenschaftlicher Exzellenz. Frankfurt/M.: Suhrkamp.

Münch, R., 2011: Akademischer Kapitalismus. Über die politische Ökonomie der Hochschulreform. Frankfurt/M.: Suhrkamp.

Nederhof, A.J., 2006: Bibliometric Monitoring of Research Performance in the Social Sciences and the Humanities: A Review. Scientometrics 66: 81-100.

Nida-Rümelin, J., 2005: Zur Zukunft der Geisteswissenschaften. Eine humanistische Perspektive. S. 11-27 in: U. Arnswald (Hrsg.), Die Zukunft der Geisteswissenschaften. Heidelberg: Manutius Verlag.

Nietzsche, F., 1954 (zuerst 1872): Über die Zukunft unserer Bildungs-Anstalten. S. 177-263 in: F. Nietzsche (Hrsg.), Werke in drei Bänden, Bd. 3. München: Carl Hanser.

Nowotny, H., 2005: The Increase of Complexity and its Reduction: Emergent Interfaces between the Natural Sciences, Humanities and Social Sciences. Theory, Culture \& Society 22: 15-31.

Nussbaum, M.C., 2010: Not for Profit: Why Democracy Needs the Humanities. Princeton: Princeton University Press.

Plumb, J.H. (Hrsg.), 1964: Crisis in the Humanities. Harmondsworth: Penguin Books.

Readings, B., 1999: The University in Ruins. Cambridge, MA, London: Harvard University Press. 
These are proofs of the final publication that is available at de Gruyter/Lucius \& Lucius via Zeitschrift für Soziologie, http://www.zfs-online.org/index.php/zfs/article/viewFile/3208/2745

Real, L.A., 2012: Collaboration in the Sciences and the Humanities. A Comparative Phenomenology. Arts and Humanities in Higher Education 11: 250-261.

Reinalter, H. (Hrsg.), 2011: Krise der Geisteswissenschaften? Ihre Bedeutung und gesellschaftliche Relevanz heute. Weimar: VDG.

Ringer, F.K., 1989: Bildung: The Social and Ideological Context of the German Historical Tradition. History of European Ideas 10: 193-202.

Ringer, F.K., 1990: The decline of the German mandarins: the German academic community, 1890-1933. Lebanon, New England: University Press of New England.

Ringer, F.K., 2004: Die Zulassung zur Universität. S. 199-226 in: W. Rüegg (Hrsg.), Geschichte der Universität in Europa, Bd. III: Vom 19. Jahrhundert zum zweiten Weltkrieg (1800-1945). München: C.H. Beck.

Ritter, J., 1974 (zuerst 1961): Die Aufgabe der Geisteswissenschaften in der modernen Gesellschaft. S. 105-140 in: J. Ritter (Hrsg.), Subjektivität. Sechs Aufsätze. Frankfurt/M.: Suhrkamp.

Rüsen, J., 2005: Plädoyer für die Geisteswissenschaften. S. 239-242 in: Kulturwissenschaftliches Institut (Hrsg.), Jahrbuch 2004. Bielefeld: transcript.

Rüsen, J., 2007: Orientierung, Bildung, Globalisierung - Plädoyer für einen geisteswissenschaftlichen Humanismus. S. 399-404 in: G. Rüther \& J.-D. Gauger (Hrsg.), Warum die Geisteswissenschaften Zukunft haben! Ein Beitrag zum Wissenschaftsjahr 2007. Freiburg: Herder.

Sapiro, G., 2003: Forms of Politicization in the French Literary Field. Theory and Society 32: 633-652.

Sarton, G., 1953: Science versus the Humanities: The History of Science. Jerusalem: Turim Press.

Scheler, M., 1954 (zuerst 1925): Die Formen des Wissens und der Bildung. S. 16-48 in: M. Scheler (Hrsg.), Philosophische Weltanschauung. München: Lehnen.

Schelling, F.W., 1830 (zuerst 1803): Vorlesungen über die Methode des akademischen Studiums. Stuttgart, Tübingen: J.G. Cotta'sche Buchhandlung.

Schlegel, A.W., 1971: Vorlesungen über das akademische Studium. Heidelberg: Lothar Stiehm.

Schleiermacher, F., 1956 (zuerst 1808): Gelegentliche Gedanken über Universitäten im deutschen Sinn, nebst einem Anhang über neu zu errichtende. S. 219-293 in: E. Anrich (Hrsg.), Die Idee der deutschen Universität. Die fünf Grundschriften aus der Zeit ihrer Neubegründung durch klassischen Idealismus und romantischen Realismus. Darmstadt: Wissenschaftliche Buchgesellschaft.

Schmidt-Wellenburg, C., 2013: Die Regierung des Unternehmens. Managementberatung im neoliberalen Kapitalismus. Konstanz: UVK.

Schnädelbach, H., 1988: Kritik der Kompensation. S. 35-45 in: K.M. Michel \& T. Spengler (Hrsg.), Kursbuch 91: Wozu Geisteswissenschaften? Berlin: Kursbuch Verlag.

Schnädelbach, H., 1990: Deutsche Philosophie seit 1945. S. 403-418 in: W. Prinz \& P. Weingart (Hrsg.), Die sog. Geisteswissenschaften: Innenansichten. Frankfurt/M.: Suhrkamp.

Shumway, D.R., 1994: Creating American Civilization: A Genealogy of American Literature as an Academic Discipline. Minneapolis: University of Minnesota Press.

Simon, D., 1989: Zukunft und Selbstverständnis der Geisteswissenschaften. Journal for History of Law 1989: 210230.

Slaughter, S. \& L.L. Leslie, 1999: Academic Capitalism: Politics, Policies, and the Entrepreneurial University. Baltimore: John Hopkins University Press.

Spranger, E., 1909: Wilhelm von Humboldt und die Humanitätsidee. Berlin: Verlag von Reuther \& Reichard.

Spranger, E., 1961 (zuerst 1953): Forschung, Berufsbildung und Menschenbildung in der gegenwärtigen deutschen Universität. S. 87-115 in: E. Spranger (Hrsg.), Kulturfragen der Gegenwart. Heidelberg: Quelle \& Meyer.

Statistisches Bundesamt, 1955: Statistisches Jahrbuch für die Bundesrepublik Deutschland. Stuttgart, Köln: W. Kohlhammer.

Statistisches Bundesamt, 1980: Statistisches Jahrbuch für die Bundesrepublik Deutschland. Stuttgart, Mainz: W. Kohlhammer.

Steenblock, V., 1999: Theorie der kulturellen Bildung. Zur Philosophie und Didaktik der Geisteswissenschaften. München: Wilhelm Fink.

Steffens, H., 1910 (zuerst 1809): Über die Idee der Universitäten. S. 205-280 in: E. Spranger (Hrsg.), Fichte, Schleiermacher, Steffens über das Wesen der Universität. Leipzig: Verlag der Dürr'schen Buchhandlung.

Steinmetz, G. (Hrsg.), 2005: The Politics of Method in the Human Sciences. Positivism and its Epistemological Others. Durham, NC: Duke University Press.

Stichweh, R., 1994: Wissenschaft. Universität. Professionen. Soziologische Analysen. Frankfurt/M.: Suhrkamp.

Strauss, A.L. \& J.M. Corbin, 1996: Grounded Theory: Grundlagen qualitativer Sozialforschung. Weinheim: Beltz.

Teichler, U., 2011: Der Jargon der Nützlichkeit. Zur Employability-Diskussion im Bologna-Prozess. S. 165-186 in: B. Hölscher \& J. Suchanek (Hrsg.), Wissenschaft und Hochschulbildung im Kontext von Wirtschaft und Medien. Wiesbaden: VS.

Titze, H., 1987: Das Hochschulstudium in Preußen und Deutschland 1820-1944. Datenhandbuch zur deutschen Bildungsgeschichte, Bd. 1,1. Göttingen: Vandenhoeck \& Ruprecht.

Torka, M., 2009: Die Projektförmigkeit der Forschung. Baden-Baden: Nomos. 
These are proofs of the final publication that is available at de Gruyter/Lucius \& Lucius via Zeitschrift für Soziologie, http://www.zfs-online.org/index.php/zfs/article/viewFile/3208/2745

Turner, S.R., 1980: The Prussian Universities and the Concept of Research. S. 68-93 in: G. Jäger, A. Martino \& F. Sengle (Hrsg.), Internationales Archiv für Sozialgeschichte der deutschen Literatur, Bd. 5. Tübingen: Max Niemeyer Verlag.

Weber, M., 1922 (zuerst 1919): Wissenschaft als Beruf. S. 524-555 in: M. Weber (Hrsg.), Gesammelte Aufsätze zur Wissenschaftslehre. Tübingen: J.C.B. Mohr.

Wehler, H.-U., 1980: Historische Sozialwissenschaft und Geschichtsschreibung. Studien zu Aufgaben und Traditionen deutscher Geschichtswissenschaft. Göttingen: Vandenhoeck \& Ruprecht.

Weingart, P., W. Prinz, M. Kastner, S. Maasen \& W. Walter, 1991: Die sog. Geisteswissenschaften: Außenansichten. Frankfurt/M.: Suhrkamp.

Wilamowitz-Moellendorff, U.v., o.J. (zuerst 1928): Erinnerungen 1848-1914. Leipzig: K.F. Koehler.

Wissenschaftsrat, 1985: Empfehlungen zum Wettbewerb im deutschen Hochschulsystem. Köln.

Zeller, E., 1879: Ueber akademisches Lehren und Lernen. Berlin: Buchdruckerei der Königlichen Akademie der Wissenschaften. 